Pacific Journal of Mathematics

ON SINGULARITY OF HARMONIC MEASURE IN SPACE 


\title{
ON SINGULARITY OF HARMONIC MEASURE IN SPACE
}

\author{
JANG-MEI WU
}

\begin{abstract}
We construct a topological ball $D$ in $\mathbf{R}^{3}$, and a set $E$ on $\partial D$ lying on a 2-dimensional hyperplane so that $E$ has Hausdorf dimension one and has positive harmonic measure with respect to $D$. This shows that a theorem of Øksendal on harmonic measure in $R^{2}$ is not true in $R^{3}$. Suppose $D$ is a bounded domain in $\mathbf{R}^{m}, m \geq 2, \mathbf{R}^{m} \backslash D$ satisfies the corkscrew condition at each point on $\partial D$; and $E$ is a set on $\partial D$ lying also on a $\mathrm{BMO}_{1}$ surface, which is more general than a hyperplane; then we can prove that if $E$ has $m-1$ dimensional Hausdorff measure zero then it must have harmonic measure zero with respect to $D$.
\end{abstract}

Lavrentiev (1936) found a simply-connected domain $D$ in $\mathbf{R}^{2}$ and a set $E$ on $\partial D$ which has zero linear measure and positive harmonic measure with respect to $D$ [5]. McMillan and Piranian subsequently simplified the example [6]. See also [1] and [3].

In [7], Øksendal proved that if $D$ is a simply-connected domain in $\mathbf{R}^{2}$, and $E$ is a set on $\partial D$ with vanishing linear measure, and if $E$ is situated on a line, then $E$ has vanishing harmonic measure $\omega(E, D)$ with respect to $D$. In [3], Kaufman and Wu generalized this result and proved that the theorem still holds if $E$ is situated on a quasi-smooth curve, but no longer holds if $E$ is situated on a quasi-conformal circle. An interesting, perhaps very difficult, question is whether the theorem is true if $E$ lies on a rectifiable curve.

Another question is the higher dimensional generalization: if $D$ is a topological ball in $\mathbf{R}^{m}, m \geq 3$, and $E$ is a set on $\partial D$, situated also on an $m-1$ dimensional hyperplane, does the vanishing of the $m-1$ dimensional Hausdorff measure, $\Lambda^{m-1}(E)=0$, imply that $\omega(E, D)=0$ ?

We answer this negatively by giving the following example.

ExAMPLE. There exists a topological ball $D$ in $\mathbf{R}^{3}$, and a set $E$ on $\partial D$, lying on a 2-dimensional hyperplane so that $E$ has Hausdorff dimension one but has positive harmonic measure with respect to $D$.

We notice that $\operatorname{dim} E=1$ is much stronger than $\Lambda^{2}(E)=0$; and that 1 is best possible, because if $\operatorname{dim} E<1$ then $E$ has zero capacity in $\mathbf{R}^{3}$, hence $E$ has zero harmonic measure with respect to $D$ in $\mathbf{R}^{3}$. 
Also this example suggests that a question left open in [1] by Carleson has no analogue in higher dimensions: if $E$ is a set on the boundary of a Jordan domain $D$, and $\Lambda^{\beta}(E)=0$ for some $1 / 2<\beta<1$, is it true that $\omega(E, D)=0$ ?

The real reason behind the example is that the Carleman-Milloux type estimation of harmonic measure is no longer valid on the boundary of a topological ball in $\mathbf{R}^{3}$. In order to obtain positive results we require the complement of the domain to be "big" near each boundary point, and allow $E$ to lie on a surface more general than a hyperplane.

Theorem. Suppose $D$ is a bounded domain in $\mathbf{R}^{m}, m \geq 2$, whose complement $\mathbf{R}^{m} \backslash D$ satisfies the corkscrew condition. Let $\Gamma$ be a topological sphere in $\mathbf{R}^{m}$, whose interior $\Omega_{1}$ and exterior $\Omega_{2}$ are both NTA domains, and on $\Gamma$,

$$
\Lambda^{m-1}(E)=0 \Rightarrow \omega\left(E, \Omega_{i}\right)=0 \quad \text { for } i=1 \text { and } 2 .
$$

Then a set on $\partial D \cap \Gamma$ having zero $\Lambda^{m-1}$ measure must have zero harmonic measure with respect to $D$.

The definitions of corkscrew condition and NTA domain are introduced by Jerison and Kenig in [2] and are given below.

Examples of $\Gamma$ that satisfy the conditions in Theorem 2 are quasismooth curves $(m=2)$ and boundaries of $\mathrm{BMO}_{1}$ domains $(m \geq 3)$; $\mathrm{BMO}_{1}$ domains are domains whose boundaries are given locally as the graph of a function $\phi$ with $\nabla \phi \in \mathrm{BMO}$, see [2] for more discussions. In these examples, the harmonic measures $\omega_{i}$ on $\Gamma$ and $\Lambda^{m-1}$ are mutually absolutely continuous, in fact, $\omega_{i} \in A_{\infty}\left(\Lambda^{m-1}\right)$.

When $m=2$, the theorem by Kaufman and Wu [3] mentioned before is not comparable to Theorem 2. There, $D$ is only simple-connected; however, $\Gamma$ has a stronger property, namely, quasi-smooth.

From the Example, we see that the corkscrew condition on $\mathbf{R}^{m} \backslash D$ cannot be discarded even when $D$ is a topological ball. Also condition (0.1) is necessasry as one can see in the case $D=\Omega_{1}$ or $\Omega_{2}$. However, we do not know whether the geometric condition on $\Gamma: \Omega_{i}$ are NTA domains, can be weakened, or whether $\Gamma$ can be replaced by a simple rectifiable curve in $\mathbf{R}^{2}$.

1. An example. We call $D$ a topological ball in $\mathbf{R}^{m}$ if it is the image of a ball under a homeomorphism of $R^{m}$. And the boundary of a topological ball is called topological sphere. For $A \in \mathbf{R}^{m}, r>0$, we let $B(A, r)=\left\{P \in \mathbf{R}^{m}:|A-P|<r\right\}$. 
For a domain $D$ in $\mathbf{R}^{m}, E \subseteq \partial D$, we denote by $\omega^{X}(E, D)$ the harmonic measure of $E$ at $X$ with respect to $D$.

LeMMA 1. In $\mathbf{R}^{2}$, there exists a simply-connected Jordan domain $\Omega$, satisfying

(1) $\Omega \cap\left\{x: x_{1}>0\right\} \subseteq\{x:|x|<2\}$

$\Omega \cap\left\{x: x_{1}<0\right\}=\left\{x: x_{1}<0,|x|<3\right\}$;

(2) $\partial_{2} \Omega$ has Hausdorff dimension 1 ;

(3) $\operatorname{cap}_{3}\left(\partial_{2} \Omega\right)>0$;

(4) $\operatorname{cap}_{3}\left(\Omega_{\varepsilon}\right) \rightarrow 0$ as $\varepsilon \rightarrow 0$;

where $\Omega_{\varepsilon}=\{x \in \Omega$ : $\operatorname{dist}(x, \partial \Omega)<\varepsilon\}, \partial_{2} \Omega$ is the boundary of $\Omega$ relative to $\mathbf{R}^{2}$, and cap $_{3}$ is the capacity with respect to the kernel $1 /|x|$.

Lemma 1 is proved at the end of this section; some readers may prefer to supply their own construction. The next lemma is the key to our example.

LeMMA 2. Let $\Omega$ be a domain in $\mathbf{R}^{2}$ with all the properties in Lemma 1 . We identify it with the set $\{(x, 0): x \in \Omega\}$ in $\mathbf{R}^{3}$. Then

$$
\omega\left(\partial_{2} \Omega, B(0,20) \backslash \bar{\Omega}\right)>0 .
$$

Proof. Choose $\varepsilon_{0}>0$ so that

$$
\operatorname{cap}_{3}\left(\Omega_{2 \varepsilon_{0}}\right)<\frac{1}{100} \operatorname{cap}_{3}\left(\partial_{2} \Omega\right) .
$$

Let $\Omega_{\varepsilon_{0}, \eta}=\Omega_{\varepsilon_{0}} \backslash \bar{\Omega}_{\eta}$, for $0<\eta<\varepsilon_{0}$, let $\mu$ and $\nu$ be the capacitary measures corresponding to $\partial_{2} \Omega$ and $\bar{\Omega}_{\varepsilon_{0}, \eta}$, with respect to the kernel $1 /|x|$, respectively. Let $U$ and $V$ be the corresponding equilibrium potentials:

$$
\begin{aligned}
& U(x)=\int_{\partial_{2} \Omega} \frac{1}{|x-y|} d \mu(y), \\
& V(x)=\int_{\bar{\Omega}_{\varepsilon_{0}, \eta}} \frac{1}{|x-y|} d \nu(y) .
\end{aligned}
$$

We recall from [4] that $U$ and $V$ are positive superharmonic on $\mathbf{R}^{3}$ bounded by 1 and are harmonic off the supports of their respective capacitary measures; moreover $U=1$ on $\partial_{2} \Omega$ except possibly on a set $S$ with $\operatorname{cap}_{3}(S)=0$ and $V=1$ on $\bar{\Omega}_{\varepsilon_{0}, \eta}$ except possibly on a set $T$ with $\operatorname{cap}_{3}(T)=0 ; \mu\left(\partial_{2} \Omega\right)=\operatorname{cap}_{3}\left(\partial_{2} \Omega\right)$ and $\nu\left(\bar{\Omega}_{\varepsilon_{0}, \eta}\right)=\operatorname{cap}_{3}\left(\bar{\Omega}_{\varepsilon_{0}, \eta}\right)$. 
Let $u=\omega\left(\partial_{2} \Omega, B(0,20) \backslash \partial_{2} \Omega\right)$ and $v=\omega\left(\bar{\Omega}_{\varepsilon_{0}, \eta}, B(0,20) \backslash \bar{\Omega}_{\varepsilon_{0}, \eta}\right)$. We observe from the last paragraph that

$$
u(X) \geq U(X)-\int_{|Y|=20} U(Y) d \omega^{X}(Y, B(0,20))
$$

for $X \in B(0,20) \backslash \partial_{2} \Omega$; and clearly $U \geq u$ and $V \geq v$ in their common domains.

For $6 \leq|X| \leq 20$ it follows from Lemma 1, (1.1), (1.2) and (1.3) that

$$
\begin{aligned}
V(X) & \leq \frac{1}{3} \operatorname{cap}_{3}\left(\bar{\Omega}_{\varepsilon_{0}, \eta}\right)<\frac{1}{300} \operatorname{cap}_{3}\left(\partial_{2} \Omega\right) \\
& <\frac{23}{300} U(X)<\frac{1}{10} U(X)
\end{aligned}
$$

for $|X|=6$, it follows from (1.2), (1.4) and (1.5) that

$$
\begin{aligned}
u(X) & \geq \frac{1}{3} U(X)+\frac{2}{3} U(X)-\frac{1}{17} \operatorname{cap}_{3}\left(\partial_{2} \Omega\right) \\
& \geq \frac{10}{3} V(X)+\frac{2}{27} \operatorname{cap}_{3}\left(\partial_{2} \Omega\right)-\frac{1}{17} \operatorname{cap}_{3}\left(\partial_{2} \Omega\right)>3 v(X) .
\end{aligned}
$$

From the maximum principle, it follows that for $|X|=6$ and $0<\eta<\varepsilon_{0}$,

$$
\begin{aligned}
\omega^{X}\left(\partial_{2} \Omega, B(0,20) \backslash\left(\bar{\Omega}_{\varepsilon_{0}, \eta} \cup \partial_{2} \Omega\right)\right) & >u-v(X)>\frac{2}{3} u(X) \\
& >\frac{1}{100} \operatorname{cap}_{3}\left(\partial_{2} \Omega\right)>0
\end{aligned}
$$

by the estimation in (1.6).

From (1.7) and the maximum principle, we obtain for $|X|=6$,

$$
\begin{gathered}
\omega^{X}\left(\partial_{2} \Omega, B(0,20) \backslash \bar{\Omega}_{\varepsilon_{0}}\right)=\inf _{0<\eta<\varepsilon_{0}} \omega^{X}\left(\Omega_{\eta} \cup \partial_{2} \Omega, B(0,20) \backslash \bar{\Omega}_{\varepsilon_{0}}\right) \\
\geq \inf _{0<\eta<\varepsilon_{0}} \omega^{X}\left(\partial_{2} \Omega, B(0,20) \backslash\left(\bar{\Omega}_{\varepsilon_{0}, \eta / 2} \cup \partial_{2}(\Omega)\right)\right) \\
>\frac{1}{100} \operatorname{cap}_{3}\left(\partial_{2} \Omega\right)>0 .
\end{gathered}
$$

Let $\alpha=\sup \left\{\omega^{X}\left(\partial_{2} \Omega, B(0,20) \backslash \bar{\Omega}_{\varepsilon_{0}}\right): x \in \Omega \backslash \Omega_{\varepsilon_{0}}\right\}$. Because $\Omega \backslash \Omega_{\varepsilon_{0}}$ has positive distance from $\partial_{2} \Omega$, we have $0<\alpha<1$. Choose $\beta$, $\alpha<\beta<1$, and a point $P$ in $B(0,20) \backslash \bar{\Omega}_{\varepsilon_{0}}$ so that $\omega^{P}\left(\partial_{2} \Omega, B(0,20) \backslash \bar{\Omega}_{\varepsilon_{0}}\right)>\beta$. By the maximum principle,

$$
\omega^{P}\left(\partial_{2} \Omega, B(0,20) \backslash \bar{\Omega}\right) \geq \omega^{P}\left(\partial_{2} \Omega, B(0,20) \backslash \bar{\Omega}_{\varepsilon_{0}}\right)-\alpha>\beta-\alpha>0 .
$$

This completes the proof. 
LeMMA 3. Let $\Omega$ be the domain in Lemma 1. Let $g(x)$ be a strictly positive continuous function on $\Omega$, defined by

$$
g(x)=\frac{1}{4} \operatorname{dist}\left(x, \partial_{2} \Omega\right) .
$$

Let

$$
G=\left\{\left(x_{1}, x_{2}, x_{3}\right):\left(x_{1}, x_{2}\right) \in \Omega \text { and }\left|x_{3}\right|<g\left(x_{1}, x_{2}\right)\right\} \text {. }
$$

Then

$$
\omega\left(\partial_{2} \Omega, B(0,20) \backslash \bar{G}\right)>0 .
$$

Proof. Suppose otherwise, we have

$$
\omega\left(\partial_{2} \Omega, B(0,20) \backslash \bar{G}\right)=0 .
$$

Let $X \in \bar{G} \backslash \bar{\Omega}, \Delta_{X}$ be the disk on $\left\{x_{3}=0\right\}$ with center $\left(X_{1}, X_{2}, 0\right)$ and of radius $\left|X_{3}\right|$ and $B_{X}$ be the ball in $\mathbf{R}^{3}$ with center $\left(X_{1}, X_{2}, 0\right)$ and of radius $2\left|X_{3}\right|$. By (1.8) and the maximum principle, we have for $X \in \bar{G} \backslash \bar{\Omega}$,

$$
\omega^{X}\left(\partial_{2} \Omega, B(0,20) \backslash \bar{\Omega}\right) \leq \omega^{X}\left(\partial B_{X}, B_{X} \backslash \overline{\Delta(X)}\right)=C<1,
$$

where $C$ is an absolute constant. Let $A$ be any point in $B(0,20) \backslash \bar{G}$. Because of (1.9) and (1.10) we have

$$
\begin{aligned}
\omega^{A}\left(\partial_{2} \Omega, B(0,20) \backslash \bar{\Omega}\right) & \\
= & \omega^{A}\left(\partial_{2} \Omega, B(0,20) \backslash \bar{G}\right) \\
& +\int_{\partial G \backslash \partial_{2} \Omega} \omega^{X}\left(\partial_{2} \Omega, B(0,20) \backslash \bar{\Omega}\right) d \omega^{A}(X, B(0,20) \backslash \bar{G}) \\
= & 0+C<1 .
\end{aligned}
$$

From (1.10) and (1.11) we see that

$$
\omega\left(\partial_{2} \Omega, B(0,20) \backslash \bar{\Omega}\right)<C<1
$$

everywhere in $B(0,20) \backslash \bar{\Omega}$. Therefore, $\omega\left(\partial_{2} \Omega, B(0,20) \backslash \bar{\Omega}\right)=0$. This contradicts Lemma 2 and proves Lemma 3.

Finally, we let $\Omega$ and $G$ be the domains in Lemma 1 and Lemma 3,

$$
D=\left\{\left(x_{1}, x_{2}, x_{3}\right): x_{1}^{2}+x_{2}^{2}<8 \text { and }\left|x_{3}\right|<4\right\} \backslash \bar{G}
$$

and

$$
E=\partial_{2} \Omega \cap\{x:|x| \leq 2\} .
$$

From the constructions of $\Omega$ and $G$, the domain $D$ is a topological ball; from properties (1) and (2) in Lemma 1, $\operatorname{dim} E=1$ and

$$
\operatorname{cap}_{3}\left(\partial_{2} \Omega \cap\{x:|x|>2\}\right)=0 .
$$


Therefore by Lemma 3,

$$
\omega(E, B(0,20) \backslash \bar{G})>0 .
$$

Arguing as in the last paragraph of the proof of Lemma 2, we conclude

$$
\omega(E, D)>0 \text {. }
$$

Consequently all the properties of $D$ and $E$ in our example are justified.

It remains to prove Lemma 1.

Proof of Lemma 1. All line segments considered below are closed. Let $l_{0,1}$ be the line segment with end points $(0,-1)$ and $(0,1)$. Let $l_{1, m}$, $m=1,2$, be two horizontal line segments with left endpoints $\left(0,-\frac{1}{2}\right)$ and $\left(0, \frac{1}{2}\right)$ respectively and of length 1 .

Suppose $\left\{l_{n-1, m}: 1 \leq m \leq 2^{n(n-1) / 2}\right\}$ have been selected for some $n \geq 2$, so that length of $l_{n-1, m}$ is $2^{-(n-1)(n-2) / 2}$. Subdivide each $l_{n-1, m}$ into $2^{n}$ equal subintervals, each of length $2^{-1-n(n-1) / 2}$. Let $\left\{l_{n, j}: 1 \leq j \leq\right.$ $\left.2^{(n+1) n / 2}\right\}$ be horizontal (if $n$ is odd) or vertical (if $n$ is even) line segments of length $2^{-n(n-1) / 2}$, with left (if $n$ is odd) or lower (if $n$ is even) endpoints coinciding with those of the subintervals of $l_{n-1, m}$ and disjoint from any $l_{n-2, m^{\prime}}$. We notice that the distance between two disjoint line segments $l_{n, m}$ and $l_{n^{\prime}, m^{\prime}}\left(n \geq n^{\prime}\right)$ is at least $2^{-1-n(n-1) / 2}$.

Let $R_{0,1}$ be the semidisk $\left\{x: x_{1}<0,|x|<3\right\}$ in $R^{2}$. We shall attach a thin rectangle to each $l_{n, m}, n \geq 1$. Let $a_{n}=2^{-2^{3 n}}$ and consider, for $n \geq 1$, the rectangle with one side coinciding with $l_{n, m}$, two opposite sides of length $a_{n}$, and interior disjoint from any $l_{n^{\prime}, m^{\prime}}$. Let $R_{n, m}$ be the interior of this rectangle together with the open line segment $S_{n, m}$ which is the side of length $a_{n}$ and lies on some $l_{n-1, m^{\prime}}$.

Let

$$
\Omega=\bigcup_{n=0}^{\infty} \bigcup_{m=1}^{2^{n(n+1) / 2}} R_{n, m}, \quad \Omega_{N}=\bigcup_{n=0}^{N} \bigcup_{m=1}^{2^{n(n+1) / 2}} R_{n, m} .
$$

We claim that $\Omega$ is simply-connected Jordan. Using induction and the fact that

$$
\left|l_{n+1, m}\right|=2^{-(n+1) n / 2}<2^{-1-n(n-1) / 2}=\operatorname{dist}\left(l_{n, m}, l_{n, m^{\prime}}\right) \quad \text { for } m \neq m^{\prime},
$$

we see that $\Omega_{n}$ is Jordan simply-connected for each $n$. Since the distance between two disjoint $l_{n, m}$ and $l_{n^{\prime}, m^{\prime}}\left(n \geq n^{\prime}\right)$ is at least $2^{-1-n(n-1) / 2}$ and

$$
\sum_{k=n+1}^{\infty}\left|l_{k, 1}\right|<2^{-1-n(n-1) / 2}-a_{n}, \text { for } n \geq 3,
$$

it follows from the construction of $\Omega$ that $\Omega$ is simply connected Jordan. Property (1) in Lemma 1 can be verified easily. 
We claim that $\partial_{2} \Omega$ has Hausdorff dimension one. Let $\delta>0$ and $r=2^{-1-n(n-1) / 2}$, which is the distance between two disjoint $l_{n, m}$ and $l_{n, m^{\prime}}$. From the construction, we see that $\partial_{2} \Omega$ can be covered by a family of $K$ squares, each of side length $r$, and $K$ no greater than

$$
C\left(2^{n(n+1) / 2}+\sum_{k=0}^{n-1} \sum_{j=1}^{2^{(k+1) k / 2}}\left|l_{k, j}\right| / 2^{-1-n(n-1) / 2}\right) \leq C 2^{n(n+1) / 2} .
$$

Therefore the $(1+\delta)$-dimensional Hausdorff measure satisfies

$$
\Lambda^{1+\delta}\left(\partial_{2} \Omega\right) \leq C \limsup 2^{n(n+1) / 2}\left(2^{-1-n(n-1) / 2}\right)^{1+\delta},
$$

$$
n \rightarrow \infty
$$

which approaches zero as $n \rightarrow \infty$. Thus $\Lambda^{1+\delta}\left(\partial_{2} \Omega\right)=0$ for every $\delta>0$, and $\partial_{2} \Omega$ has dimension at most 1 . It is clear $\partial_{2} \Omega$ has dimension at least 1 .

Next, we claim that $\operatorname{cap}_{3}\left(\partial_{2} \Omega\right)$ is positive. Recall that $\partial_{2} \Omega$ is a Jordan curve and $S_{n, m}$ is a particular side of $R_{n, m}$ that is situated on some $l_{n-1, m^{\prime}}$. Let $A_{n, m}$ and $B_{n, m}$ be the endpoints of $S_{n, m}$; from the construction of $\Omega$, one sees that $A_{n, m}$ and $B_{n, m}$ are on $\partial_{2} \Omega$. Let $\mu$ be the probability measure on $\partial_{2} \Omega$ satisfying, for $n \geq 1$,

$$
\mu\left(E_{n, m}\right)=2^{-n(n+1) / 2},
$$

where $E_{n, m}$ is the subarc of $\partial_{2} \Omega$ with endpoints $A_{n, m}$ and $B_{n, m}$ which does not contain the point $(-3,0)$.

We shall prove that

$$
\mu\left(\partial_{2} \Omega \cap \Delta(P, t)\right) \leq C t\left(\log \frac{1}{t}\right)^{-2}
$$

for every $P \in \mathbf{R}^{2}$ and $0<t<t_{0}$. Once (1.13) is proved, we have for any $P \in \mathbf{R}^{2}$,

$$
\begin{aligned}
\int_{\partial_{2} \Omega} \frac{1}{|P-X|} d \mu(X) & =\int_{0}^{\infty} \mu\left(\Delta(P, t) \cap \partial_{2} \Omega\right) \frac{d t}{t^{2}} \\
& \leq \int_{t_{0}}^{1} \frac{d t}{t^{2}}+\int_{0}^{t_{0}} \frac{1}{t \log ^{2}(1 / t)} d t<C\left(t_{0}\right)<\infty .
\end{aligned}
$$

Therefore $\operatorname{cap}_{3}\left(\partial_{2} \Omega\right)>0$.

To prove (1.13), we assume

$$
2^{-n(n-1) / 2} \leq t<2^{-(n-1)(n-2) / 2} .
$$

For any $P \in \mathbf{R}^{2}, \Delta(P, t)$ meets at most $C t 2^{n(n-1) / 2}$ arcs of the form $E_{n, m}$. Therefore by (1.12),

$$
\begin{aligned}
\mu\left(\Delta(p, t) \cap \partial_{2} \Omega\right) & \leq C t 2^{n(n-1) / 2} 2^{-n(n+1) / 2} \\
& \leq C t 2^{-n}<C t\left(\log \frac{1}{t}\right)^{-2}
\end{aligned}
$$

if $0<t<t_{0}$. 
Finally we prove that $\operatorname{cap}_{3}\left(\Omega_{\varepsilon}\right) \rightarrow 0$ as $\varepsilon \rightarrow 0^{+}$. Because $\operatorname{cap}_{3}\left(\Omega_{\varepsilon}\right)$ decreases as $\varepsilon$ decreases, we need only to show that $\operatorname{cap}_{3}\left(\Omega_{a_{N}}\right) \rightarrow 0$ as $N \rightarrow \infty$. We observe, by the relative narrowness of $a_{N}$ to the distance between $R_{n, m}$ and $R_{n^{\prime}, m^{\prime}}\left(n, n^{\prime}<N\right)$, that

$$
\Omega_{a_{N}} \subseteq \bigcup_{n=0}^{N-1} \bigcup_{m=1}^{2^{n(n+1) / 2}} R_{n, m, a_{N}} \cup \bigcup_{n=N}^{\infty} \bigcup_{m=1}^{2^{n(n+1) / 2}} R_{n, m}
$$

where $R_{n, m, a_{N}}=\left\{x \in R_{n, m}, \operatorname{dist}\left(x, \partial R_{n, m}\right)<a_{N}\right\}$. By a variation of Lemma 4 below, we have the following estimation:

$$
\begin{aligned}
& \operatorname{cap}_{3}\left(\Omega_{a_{N}}\right) \\
& \leq C\left(\sum_{n=0}^{N-1} 2^{n(n+1) / 2} \frac{\left|l_{n, 1}\right|}{\log \left(\left|l_{n, 1}\right| / a_{N}\right)}+\sum_{n=N}^{\infty} 2^{n(n+1) / 2} \frac{\left|l_{n, 1}\right|}{\log \left(\left|l_{n, 1}\right| / a_{n}\right)}\right) \\
& \leq C\left(\sum_{n=0}^{N-1} \frac{2^{n(n+1) / 2} 2^{-n(n-1) / 2}}{\log \left(2^{-n(n-1) / 2} 2^{2^{2 N}}\right)}+\sum_{n=N}^{\infty} \frac{2^{n(n+1) / 2} 2^{-n(n-1) / 2}}{\log \left(2^{-n(n-1) / 2} 2^{2^{3 n}}\right)}\right) \\
& \leq \sum_{n=0}^{N-1} 2^{n} 2^{-2 N}+\sum_{n=N}^{\infty} 2^{-n},
\end{aligned}
$$

which approaches 0 as $N \rightarrow \infty$. This completes the proof of Lemma 1 .

LeMMA 4 [4; p. 165]. Let $E$ be an elongated ellipsoid of revolution with axes $a, b(b<a)$. Then

$$
\operatorname{cap}_{3}(E)=\frac{2}{\pi} \frac{\sqrt{a^{2}-b^{2}}}{\log \left[\left(a+\sqrt{a^{2}-b^{2}}\right) /\left(a-\sqrt{a^{2}-b^{2}}\right)\right]} .
$$

2. Proof of the Theorem. Following the definition in [2], we say a domain $\Omega$ in $\mathbf{R}^{m}$ is a non-tangentially accessible (NTA) domain if there exist fixed constants $M=M(\Omega)>10$ and $r_{0}=r_{0}(\Omega)>0$ such that the following conditions are satisfied.

(2.1) corkscrew condition: for any $Q \in \partial \Omega, r<r_{0}$, there exists $A=$ $A_{r}(Q) \in \Omega$ such that $M^{-1} r<|A-Q|<r$ and $\operatorname{dist}(A, \partial \Omega)>M^{-1} r$;

(2.2) $\mathbf{R}^{m} \backslash \Omega$ satisfies the corkscrew condition;

(2.3) Harnack chain condition: if $X_{1}$ and $X_{2} \in \Omega$, $\operatorname{dist}\left(X_{i}, \partial D\right)>\varepsilon>0$, $i=1,2$, and $\left|X_{1}-X_{2}\right| \leq K \varepsilon$, then there exist balls $B_{j}=B\left(Y_{j}, r_{j}\right), 1 \leq j$ $\leq L, L$ depending only on $K$, but not on $\varepsilon$, so that $Y_{1}=X_{1}$ and 
$Y_{L}=X_{2} ;$ and the balls $B_{j}$ satisfy

$$
M^{-1} r_{j}<\operatorname{dist}\left(B_{j}, \partial \Omega\right)<M r_{j}, \quad 1 \leq j \leq L
$$

and

$$
B\left(Y_{j}, r_{j} / 2\right) \cap B\left(Y_{j}, r_{j+1} / 2\right) \neq \varnothing, \quad 1 \leq j \leq L-1 .
$$

( $\left\{B_{j}\right\}$ is called a Harnack chain from $X_{1}$ to $X_{2}$ of length $L$.)

Assuming $F \subseteq \partial D \cap \Gamma$ and $\Lambda^{m-1}(F)=0$, we want to show $\omega(F, D)$ $=0$.

We claim that it is enough to prove that there exists $0<\beta<1$, so that

$$
\omega^{Q}(F, D)<\beta \quad \text { for every } Q \in D \cap \Gamma .
$$

In fact, for $X \in D \cap \Omega_{i}$, it follows from (0.1) that

$$
\omega^{X}\left(F, D \cap \Omega_{i}\right) \leq \omega^{X}\left(F, \Omega_{i}\right)=0 ;
$$

hence

$$
\begin{aligned}
\omega^{X}(F, D) & =\omega^{X}\left(F, D \cap \Omega_{i}\right)+\int_{\Gamma \cap D} \omega^{Q}(F, D) d^{X}\left(Q, D \cap \Omega_{i}\right) \\
& =\int_{\Gamma \cap D} \omega^{Q}(F, D) d \omega^{X}\left(Q, D \cap \Omega_{i}\right)
\end{aligned}
$$

After (2.6) is proved, we may conclude

$$
\omega^{X}(F, D)<\beta<1 \quad \text { for every } X \in D \text {. }
$$

This is possible only when $\omega(F, D)=0$. Therefore we need only to show (2.6).

Since $\Omega_{i}, \quad i=1,2$, are NTA domains and $\mathbf{R}^{m} \backslash D$ satisfies the corkscrew condition, we let

$$
M=\max \left\{M\left(\Omega_{1}\right), M\left(\Omega_{2}\right), M(D)\right\}
$$

and

$$
r_{0}=\min \left\{r_{0}\left(\Omega_{1}\right), r_{0}\left(\Omega_{2}\right), r_{0}(D)\right\}
$$

from their respective definitions.

For a fixed $Q \in D \cap \Gamma$, let

$$
r=\min \left\{r_{0}, \operatorname{dist}(Q, \partial D)\right\} .
$$

From the corkscrew condition on $\Omega_{i}$, we can find

$$
U_{i}=B\left(A_{i}, r / 4 M\right) \subseteq \Omega_{i}
$$


so that

$$
\left|A_{i}-Q\right|<r / 2 \text { and } \operatorname{dist}\left(U_{i}, \Gamma\right)>r / 4 M .
$$

Notice that $U_{1} \cup U_{2} \subseteq B(Q, r) \subseteq D$. Therefore we can find $\alpha, 0<\alpha<1$, depending on $M$ only so that

$$
\omega^{Q}(F, D) \leq 1-\alpha+\alpha \sup _{X \in \bar{U}_{i}} \omega^{X}(F, D), \quad \text { for } i=1 \text { or } 2 .
$$

Because of (2.7) and (2.9), in order to prove (2.6), we need only to show there exists $\eta<1$ so that

$$
\min \left\{\sup _{X \in \bar{U}_{t}} \omega^{X}\left(\Gamma \cap D, D \cap \Omega_{i}\right): i=1,2\right\}<\eta .
$$

We claim that there exists a ball

$$
V \equiv B\left(A,(4 M)^{-2} r\right)
$$

whose closure is completely in $\Omega_{1} \backslash D$ or completely in $\Omega_{2} \backslash D$, and

$$
|A-Q|<K r \text { and } \operatorname{dist}(V, \Gamma)>(4 M)^{-2} r,
$$

where $K=2+(\operatorname{diam} D) / r_{0}$.

In fact, let $P$ be a point on $\partial D$ so that $|P-Q|=\operatorname{dist}(Q, \partial D)$. Since $\mathbf{R}^{m} \backslash D$ satisfies the corkscrew condition, we can find a ball

$$
W=B\left(Y,(2 M)^{-1} r\right) \subseteq \mathbf{R}^{m} \backslash D
$$

so that

$$
|Y-P|<r \text { and } \operatorname{dist}(W, \partial D)>(2 M)^{-1} r .
$$

If $B\left(Y,(4 M)^{-1} r\right) \cap \Gamma=\varnothing$ then $B\left(Y,(4 M)^{-1} r\right)$ lies completely in $\Omega_{1} \backslash D$ or completely in $\Omega_{2} \backslash D$; we let

$$
A \equiv Y \quad \text { and } \quad V \equiv B\left(Y,(4 M)^{-2} r\right)
$$

and can verify (2.11) easily.

If $B\left(Y,(4 M)^{-1} r\right) \cap \Gamma$ contains some point $Z$, by the corkscrew condition on $\Omega_{1}$, we can find

$$
V \equiv B\left(A,(4 M)^{-2} r\right) \subseteq \Omega_{1}
$$

so that

$$
\left(8 M^{2}\right)^{-1} r<|A-Z|<(8 M)^{-1} r \text { and } \operatorname{dist}(V, \Gamma)>(4 M)^{-2} r .
$$


Because $|A-Y| \leq|A-Z|+|Z-Y| \leq 3 r(8 M)^{-1}$, we see $V \subseteq W \subseteq$ $\mathbf{R}^{m} \backslash D$. Therefore $V \subseteq \Omega_{1} \backslash D$. Again (2.11) can be verified easily. This proves our claim.

From now on we assume $V$ is contained in $\Omega_{1} \backslash D$, and shall prove

$$
\sup _{X \in \bar{U}_{1}} \omega^{X}\left(\Gamma \cap D, D \cap \Omega_{1}\right)<\eta<1 .
$$

When $V$ is in $\Omega_{2} \backslash D$, we argue similarly.

From (2.8) and (2.11) and the assumption that $\Omega_{1}$ is an NTA domain, we can find a Harnack chain $\left\{B_{j}\right\}_{j=1}^{L}$ in $\Omega_{1}$, whose length $L$ depends on $r_{0}, M$ and diam $D$ only, that connects $A$ to $A_{1}$; moreover, we may choose

$$
\begin{aligned}
& B_{1} \equiv B\left(A, 3 r\left(32 M^{2}\right)^{-1}\right) \supseteq B\left(A, r(4 M)^{-2}\right)=V, \\
& B_{L} \equiv B\left(A_{1}, 3 r(8 M)^{-1}\right) \supseteq B\left(A_{1}, r(4 M)^{-1}\right)=U_{1},
\end{aligned}
$$

so that (2.4) is still satisfied with a bigger constant $M^{\prime}$ dependent only on $M, r_{0}$ and $\operatorname{diam} D$.

Let $B=\bigcup_{j=1}^{L} B_{j}$ and

$$
w=\left\{\begin{array}{cl}
\omega\left(\Gamma \cap D, D \cap \Omega_{1}\right) & \text { on } D \cap \Omega_{1}, \\
0 & \text { on } \mathbf{R}^{m} \backslash\left(D \cap \Omega_{1}\right) .
\end{array}\right.
$$

Since $\left\{B_{j}\right\}$ is a Harnack chain, $\bar{B} \subseteq \Omega_{1}$; hence $w$ is subharmonic on $B$; and because $\bar{V} \cap D=\varnothing, w=0$ on $\bar{V}$. Therefore by the maximum principle, for $X \in \bar{U}_{1} \subseteq D \cap \Gamma_{1}$

$$
\omega^{X}\left(\Gamma \cap D, D \cap \Omega_{1}\right) \leq \omega^{X}(\partial B, B \backslash \bar{V}) .
$$

By (2.13), (2.14), properties (2.4) and (2.5) of the Harnack chain condition, and the Harnack principle, we can find $\eta<1$, depending on $r_{0}, M$, diam $D$, so that

$$
\omega^{X}(\partial D, B \backslash \bar{V})<\eta \quad \text { for every } X \in \bar{U}_{1} .
$$

Therefore (2.12) is proved, and thus (2.6) follows.

\section{REFERENCES}

[1] L. Carleson, On the distortion of sets on a Jordan curve under conformal mapping, Duke Math. J., 40 (1973), 547-559.

[2] D. S. Jerison and C. E. Kenig, Boundary behavior of harmonic functions in non-tangentially accessible domains, Advances in Math., 46 (1982), 80-147.

[3] R. Kaufman and J.-M. Wu, Distortion of the boundary under conformal mapping, Michigan Math. J., 29 (1982), 267-280. 
[4] N. S. Landkof, Foundations of Modern Potential Theory, (1965), (Russian), SpringerVerlag (1972).

[5] M. A. Lavrentiev, Boundary problems in the theory of univalent functions, Mat. Sb., 43 (1936), 815-846; (Russian), Amer. Math. Soc. Transl., 32 (1963), 1-35.

[6] J. McMillan and G. Piranian, Compression and expansion of boundary sets, Duke Math. J., 40 (1973), 599-605.

[7] B. Øksendal, Brownian motion and sets of harmonic measure zero, Pacific J. Math., 95 (1981), 193-204.

Received February 18, 1984. Research partially supported by the National Science Foundation.

UNIVERSITY OF ILLINOIS

URBANA, IL 61801 


\title{
PACIFIC JOURNAL OF MATHEMATICS EDITORS
}

V. S. VARADARAJAN (Managing Editor)
University of California
Los Angeles, CA 90024
HEBERT ClEMENS
University of Utah
Salt Lake City, UT 84112
CHARLES R. DEPRIMA
California Institute of Technology
Pasadena, CA 91125

R. FINN

Stanford University

Stanford, CA 94305

HERMANN FLASCHKA

University of Arizona

Tucson, AZ 85721

Ramesh A. Gangolli

University of Washington

Seattle, WA 98195

ROBION KIRBY

University of California

Berkeley, CA 94720
C. C. MOORE

University of California

Berkeley, CA 94720

H. SAMELSON

Stanford University

Stanford, CA 94305

HaRold Stark

University of California, San Diego

La Jolla, CA 92093

\section{ASSOCIATE EDITORS}

\author{
R. ARENS \\ E. F. BECKENBACH \\ B. H. NeumanN \\ F. Wolf \\ K. YoSHIDA \\ (1906-1982)
}

\section{SUPPORTING INSTITUTIONS}

UNIVERSITY OF ARIZONA
UNIVERSITY OF BRITISH COLUMBIA
CALIFORNIA INSTITUTE OF TECHNOLOGY
UNIVERSITY OF CALIFORNIA
MONTANA STATE UNIVERSITY
UNIVERSITY OF NEVADA, RENO
NEW MEXICO STATE UNIVERSITY
OREGON STATE UNIVERSITY

UNIVERSITY OF OREGON

UNIVERSITY OF SOUTHERN CALIFORNIA

STANFORD UNIVERSITY

UNIVERSITY OF HAWAII

UNIVERSITY OF TOKYO

UNIVERSITY OF UTAH

WASHINGTON STATE UNIVERSITY UNIVERSITY OF WASHINGTON

The Supporting Institutions listed above contribute to the cost of publication of this Journal, but they are not owners or publishers and have no responsibility for its content or policies.

Mathematical papers intended for publication in the Pacific Journal of Mathematics should be in typed form or offset-reproduced (not dittoed), double spaced with large margins. Please do not use built up fractions in the text of the manuscript. However, you may use them in the displayed equations. Underline Greek letters in red, German in green, and script in blue. The first paragraph must be capable of being used separately as a synopsis of the entire paper. In particular it should contain no bibliographic references. Please propose a heading for the odd numbered pages of less than 35 characters. Manuscripts, in triplicate, may be sent to any one of the editors. Please classify according to the scheme of Math. Reviews, Index to Vol. 39. Supply name and address of author to whom proofs should be sent. All other communications should be addressed to the managing editor, or Elaine Barth, University of California, Los Angeles, California 90024.

There are page-charges associated with articles appearing in the Pacific Journal of Mathematics. These charges are expected to be paid by the author's University, Government Agency or Company. If the author or authors do not have access to such Institutional support these charges are waived. Single authors will receive 50 free reprints; joint authors will receive a total of 100 free reprints. Additional copies may be obtained at cost in multiples of 50 .

The Pacific Journal of Mathematics is issued monthly as of January 1966. Regular subscription rate: $\$ 190.00$ a year (5 Vols., 10 issues). Special rate: $\$ 95.00$ a year to individual members of supporting institutions.

Subscriptions, orders for numbers issued in the last three calendar years, and changes of address should be sent to Pacific Journal of Mathematics, P.O. Box 969, Carmel Valley, CA 93924, U.S.A. Old back numbers obtainable from Kraus Periodicals Co., Route 100, Millwood, NY 10546.

The Pacific Journal of Mathematics at P.O. Box 969, Carmel Valley, CA 93924 (ISSN 0030-8730) publishes 5 volumes per year. Application to mail at Second-class postage rates is pending at Carmel Valley, California, and additional mailing offices. Postmaster: Send address changes to Pacific Journal of Mathematics, P.O. Box 969, Carmel Valley, CA 93924.

\section{PUBLISHED BY PACIFIC JOURNAL OF MATHEMATICS, A NON-PROFIT CORPORATION}

Copyright $\subset 1986$ by Pacific Journal of Mathematics 


\section{Pacific Journal of Mathematics}

\section{Vol. 121, No. 2 December, 1986}

Jorge Almeida, Minimal nonpermutative pseudovarieties of semigroups.

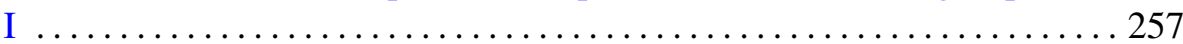

Jorge Almeida, Minimal nonpermutative pseudovarieties of semigroups.

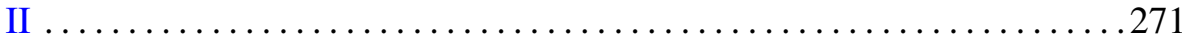

Carlos Andradas Heranz and José Manuel Gamboa Mutuberría, On

projections of real algebraic varieties $\ldots \ldots \ldots \ldots \ldots \ldots \ldots \ldots \ldots 281$

Zeev Ditzian, Inverse theorems for multidimensional Bernstein operators . . 293

M. Furi and Maria Patrizia Pera, A continuation principle for forced oscillations on differentiable manifolds $\ldots \ldots \ldots \ldots \ldots \ldots \ldots \ldots \ldots . \ldots . \ldots . \ldots 321$

James J. Hebda, The collars of a Riemannian manifold and stable

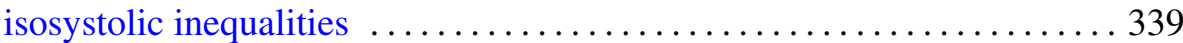

Henryk Hecht and Dragan Miličić, Character identities and asymptotic behavior of matrix coefficients of discrete series ................ 357

Piotr Jakóbczak, The boundary regularity of the solution of the $\bar{\partial}$-equation in the product of strictly pseudoconvex domains $\ldots \ldots \ldots \ldots \ldots \ldots . \ldots 371$

Krzysztof Jarosz, Isometries between injective tensor products of Banach

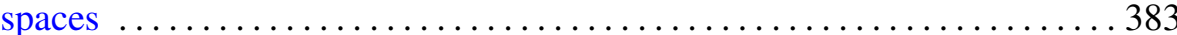

Hans Keller, On valued, complete fields and their automorphisms ........ 397

David Masser and Peter Man-Kit Shiu, On sparsely totient numbers . . . . 407

Tze-Beng Ng, Vector bundles over $(8 k+3)$-dimensional manifolds

Thomas Joseph Ransford, The spectrum of an interpolated operator and analytic multivalued functions

Akihito Uchiyama, On the radial maximal function of distributions 467 Jang-Mei Gloria Wu, On singularity of harmonic measure in space 